\title{
EBV-Associated Post-Transplantation B-Cell Lymphoproliferative Disorder in Patient after Allogenic Stem Cell Transplantation
}

\author{
Li Tang1, Yu Li ${ }^{*}$ \\ ${ }^{1}$ Department of Pathophysiology, Chongqing Medical University, Chongqing, China \\ ${ }^{2}$ Department of Pathology, Institute of Neuroscience, Chongqing Medical University, Chongqing, China \\ Email: tangli@cqmu.edu.cn, *liyu@cqmu.edu.cn
}

How to cite this paper: Tang, L. and Li, Y. (2019) EBV-Associated Post-Transplantation B-Cell Lymphoproliferative Disorder in Patient after Allogenic Stem Cell Transplantation. Journal of Biosciences and Medicines, 7, 11-15.

https://doi.org/10.4236/jbm.2019.75003

Received: February 26, 2019

Accepted: May 14, 2019

Published: May 17, 2019

\begin{abstract}
Epstein-Barr virus (EBV)-associated B-cell post-transplantation lymphoproliferative disorder (PTLD) is a severe complication following solid-organ transplantation (SOT) and allogeneic hematopoietic stem cell transplantation (HSCT). We present a case of a 15-year-old male developing a monomorphic B-cell PTLD after receiving an allogenic stem cell transplant for acute acute myeloid leukemia. A diagnostic lymph node biopsy revealed monomorphic type, B cell phenotype, associated with Epstein-Barr virus, consistent with post-transplant lymphoproliferative disorder (PTLD). The morbidity and mortality of PTLD are high, and there is no standard protocol for treatment of PTLD. To prevent the occurrence of PTLD and early intervention are important for the prognosis of patients.
\end{abstract}

\section{Keywords}

Post-Transplantation Lymphoproliferative Disorder, Allogeneic

Hematopoietic Stem Cell Transplantation, Epstein-Barr Virus

\section{Introduction}

Post-transplantation lymphoproliferative disorder (PTLD) is a serious complication following solid-organ transplantation (SOT) and allogeneic hematopoietic stem cell transplantation (HSCT) [1]. It represents a spectrum of lymphoid proliferations which occur in the setting of immuno-suppression and organ transplantation. It is also generally an Epstein-Barr Virus (EBV) associated proliferation and is mostly of B-cell origin [2]. EBV-positive PTLD typically occurs relatively early, the highest incidence presences in the first year after trans-plant [3]. 
The incidence of PTLD varies with the transplanted organ and the morbidity and mortality of PTLD are high. There is no standard protocol for treatment of PTLD. Current treatment options include rituximab and chemotherapy.

\section{Materials and Methods}

\subsection{Patient}

A 15-year-old male acute myeloid leukemia $\mathrm{M} 2 \mathrm{~b}(\mathrm{ETO}+)$ patient presented with fever for 32 days after the second chemotherapy and allogeneic hematopoietic stem cell transplantation. The patient suffered from repeated infectious episodes, such as bacterial sepsis (E. coli, Acinetobacter baumannii resistant to carbapenems), Occult fungal infection, Occult virus infection and hemorrhagic cystitis. After transplantation, CT-scan showed multiple chest and abdominal lymphadenopathy and superficial lymph node enlargement.

\subsection{Methods}

Paraffin-embedded $5 \mathrm{~mm}$ thick cervical lymph node tissue sections were prepared and stained with hematoxylin-eosin for light microscopic evaluation. Paraffin section immunohistochemistry was performed to detect the expression of CD20, CD21, CD23, CD10, CD2, CD3, CD5, CD7, Bcl-2, Bcl-6, C-myc and Ki67. EBV encoded RNA (EBER) in situ hybridization studie was performed according to the manufacturer's protocol. Clonal rearrangement of IgH and Igk gene were performed by polymerase chain reaction (PCR). Fluorescence in situ hybridization (FISH) was also used for IgH/Bcl-2 and C-myc gene detection.

\section{Results}

The PTLD had morphologic features of a large cell lymphoma. Immunohistochemistry studies demonstrated a B cell phenotype in the large lymphocytes exhibited by positive immunoreactivity for CD20, CD21 and CD23 did not show follicular dendritic cell (FDC) network (Figure 1). Focally B-cell lymphoma (Bcl)-2 (60\%+), C-myc (30\%+), the proportion of Ki67-positive cells was $90 \%$. CD10-, Bcl6-, multiple myeloma oncogene (MUM)-1 (+) (Figure 2). The expression of $\mathrm{T}$ cell markers $\mathrm{CD} 2, \mathrm{CD} 3, \mathrm{CD} 5$ and $\mathrm{CD} 7$ were partly positive. EBER

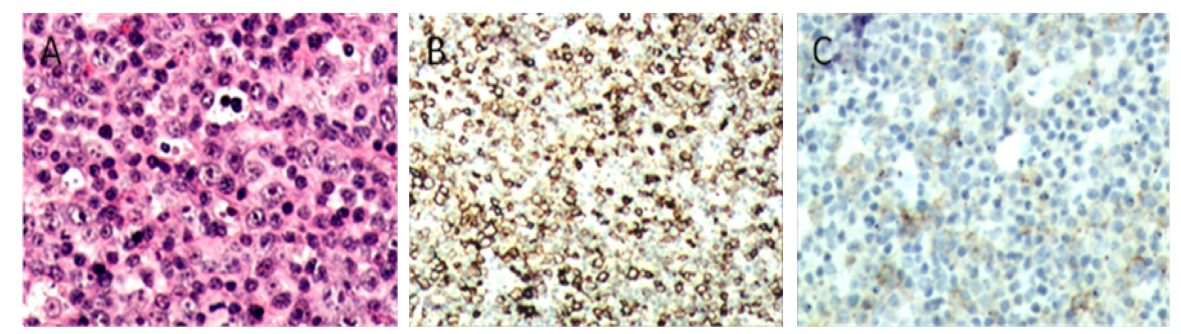

Figure 1. (A) Hematoxylin and eosin (H \& E) staining of histopathology $(\times 100)$. The diffused proliferation of lymphoid cells was observed. (B) Immunohistochemistry of CD20 $(\times 100)$. The staining was diffusely positive for CD20. (C) Immunohistochemistry of CD21 (×100). 

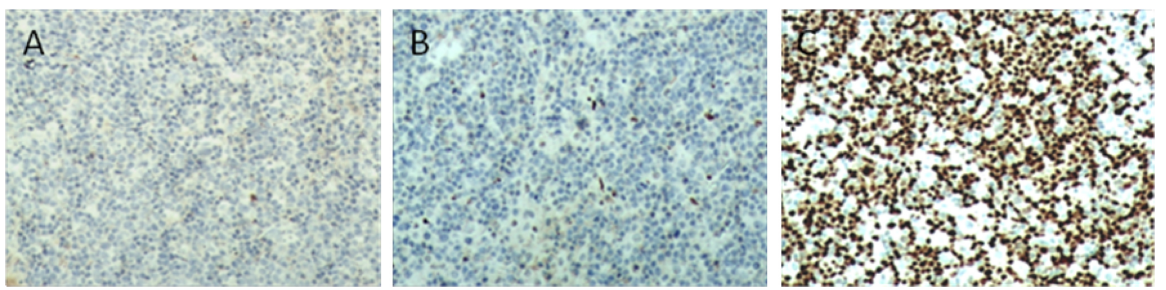

Figure 2. Immunohistochemistry of CD10 (A), Bcl6 (B), and MUM-1 (C) (×100).

was detected in the large lymphocytes by ISH and showed 200/HPF. From phenotype, possibility of lymphoma was considered. PCR analysis showed clonal rearrangement of IgH gene and Igk gene in B cells and clonal rearrangement of $\mathrm{T}$ cell TCR gamma gene. FISH showed negative expression of IgH/Bcl-2 and C-myc gene. Thus, PTLD, of monomorphic type and with a B cell phenotype was considered (Diffuse large B cell lymphoma, not otherwise specified, DLCBL-NOS).

\section{Discussion}

PTLD is a fatal complication of solid organ transplantation and allogeneic hematopoietic stem cell transplantation. It is often acute and disseminated, progresses rapidly, and associated with a poor prognosis [4] [5]. The main clinical manifestations are fever, lymph node enlargement, hepatosplenomegaly splenomegalyand pharyngitis [6]. PTLD mainly occurs within 6 months of transplantation, progresses rapidly and may cause multiple organ failure or even death soon after diagnosis. Immunosuppression, viral infections, recipient age and ethnicity, and allograft type are risk factors for PTLD. EBV primary infection or reactivation plays a key role in the pathogenesis. Hematopoietic stem cell transplantation patients lack of effective immunity monitoring, $\mathrm{T}$ cell function is depressed under immunosuppression. When EBV infection occurs, it is likely to result in substantial replication of EBV which causes abnormal proliferation of $B$ lymphocytes and eventually lead to the development of PTLD. But about $1 / 3$ of PTLD are EBV negative. Compared with EBV+ patients, the clinical characteristics of EBV-PTLD are different. In the updated 2016 World Health Organization lymphoma classification [7], PTLD is subclassified as plasmacytic hyperplasia PTLD, infectious mononucleosis PTLD, florid follicular hyperplasia PTLD, polymorphic PTLD, monomorphic PTLD (B- and T-/NK-cell types) or classical Hodgkin lymphoma PTLD. The typical pathology of PTLD is the presence of a large number of plasma cell-like B cells, a few T cells among lymphocytes, and focal necrosis. The current patient was diagnosed with monomorphic PTLD (B-cell type). Monomorphic PTLD is the most common category of PTLD in adult patients and diffuse large B-cell lymphoma accounts for the majority of PTLD cases. The other types are Burkitt lymphoma, plasma cell myeloma, and plasmacytoma-like lesions.

Hematopoietic stem cell transplantation (HSCT) is an effective strategy in children [8]. However, the source of hematopoietic stem cells (HSCs) may affect 
short and long-term complications. After HSCT, PTLD, especially EBV-positive PTLD may occur with current treatments. The clinical presentation is often nonspecific and tissue biopsy is necessary to establish the diagnosis. In this case, it had a B cell phenotype, the presence of EBER in lymphoid proliferation supported the diagnosis of PTLD. FISH showed negative expression of IgH/Bcl-2 and $\mathrm{C}$-myc gene. Cases that appear blastoid or cases intermediate between DLBCL and BL, but which lack a MYC and BCL2 and/or BCL6 rearrangement, will be placed in the category of high-grade B-cell lymphoma, not otherwise specified (HGBL, NOS) .

Because PTLD results from functional over-immunosuppression, the initial treatment is reduction of immunosuppression. At present there is no standardized approach to the evaluation and treatment of PTLD after transplantation. Effective treatment before multiple organ dysfunction is very important for the curative effect of PTLD. Antiviral agents, interferon, immuno-based monoclonal therapy, cell-based therapy and chemotherapy also have a potential role in treating this disorder [9] [10]. To prevent the occurrence of PTLD and early intervention are important for the prognosis of patients.

\section{Conflicts of Interest}

The authors declare no conflicts of interest regarding the publication of this paper.

\section{References}

[1] Kasahara, H., Mori, T., Kato, J., et al. (2015) Post-Transplant Lymphoproliferative Disorder of the Adrenal Gland after Allogeneic Hematopoietic Stem Cell Transplantation: Report of Two Cases and Literature Review. Transpl Infect Dis., 17, 909-914. https://doi.org/10.1111/tid.12461

[2] San-Juan, R., Comoli, P., Caillard, S., et al. (2014) Epstein-Barr Virus-Related Post-Transplant Lymphoproliferative Disorder in Solid Organ Transplant Recipients. Clin Microbiol Infect., 20, 109-118. https://doi.org/10.1111/1469-0691.12534

[3] Green, M. and Michaels, M.G. (2013) Epstein-Barr Virus Infection and Posttransplant Lymphoproliferative Disorder. Am J Transplant, 13, 41-54. https://doi.org/10.1111/ajt.12004

[4] Choi, J.H., Park, B.B., Suh, C., et al. (2010) Clinical Characteristics of Monomorphic Post-Transplant Lymphoproliferative Disorders. J Korean Med Sci, 25, 523-526. https://doi.org/10.3346/jkms.2010.25.4.523

[5] Reddy, N., Rezvani, K., Barrett, A.J., et al. (2011) Strategies to Prevent EBV Reactivation and Posttransplant Lymphoproliferative Disorders (PTLD) after Allogeneic Stem Cell Transplantation in High-Risk Patients. Biol Blood Marrow Transplant, 17, 591-597. https://doi.org/10.1016/j.bbmt.2010.08.007

[6] Nijland, M.L., Kersten, M.J., Pals, S.T., et al. (2016) Epstein-Barr Virus-Positive Posttransplant Lymphoproliferative Disease after Solid Organ Transplantation: Pathogenesis, Clinical Manifestations, Diagnosis, and Management. Transplant Direct, 2, e48. https://doi.org/10.1097/TXD.0000000000000557

[7] Swerdlow, S.H., Campo, E., Pileri, S.A., et al. (2016) The 2016 Revision of the World Health Organization Classification of Lymphoid Neoplasms. Blood., 127, 
2375-2390. https://doi.org/10.1182/blood-2016-01-643569

[8] Selvanathan, A., Ellaway, C., Wilson, C., et al. (2018) Effectiveness of Early Hematopoietic Stem Cell Transplantation in Preventing Neurocognitive Decline in $\mathrm{Mu}$ copolysaccharidosis Type II: A Case Series. JIMD Rep., 41, 81-89.

https://doi.org/10.1007/8904_2018_104

[9] Al-Mansour, Z., Nelson, B.P. and Evens, A.M. (2013) Post-Transplant Lymphoproliferative Disease (PTLD): Risk Factors, Diagnosis, and Current Treatment Strategies. Curr Hematol Malig Rep., 8, 173-183.

https://doi.org/10.1007/s11899-013-0162-5

[10] Singavi, A.K., Harrington, A.M. and Fenske, T.S. (2015) Post-Transplant Lymphoproliferative Disorders. Cancer Treat Res., 165, 305-327.

https://doi.org/10.1007/978-3-319-13150-4_13 